\title{
Image Segmentation Based on a Dynamically Coupled Neural Oscillator Network
}

\author{
Ke Chen ${ }^{\dagger \ddagger}$ and DeLiang L. Wang ${ }^{\dagger}$ \\ ${ }^{\dagger}$ Department of Computer and Information Science and Center for Cognitive Science \\ The Ohio State University, Columbus, OH 43210-1277, USA \\ ${ }^{\ddagger}$ National Laboratory of Machine Perception and Center for Information Science \\ Peking University, Beijing 100871, China \\ E-mail: chen@cis.pku.edu.cn, dwang @ cis.ohio-state.edu
}

\begin{abstract}
In this paper, a dynamically coupled neural oscillator network is proposed for image segmentation. Instead of pair-wise coupling, an ensemble of oscillators coupled in a local region is used for grouping. We introduce a set of neighborhoods to generate dynamical coupling structures associated with a specific oscillator. Based on the proximity and similarity principles, two grouping rules are proposed to explicitly consider the distinct cases of whether an oscillator is inside a homogeneous image region or near a boundary between different regions. The use of dynamical coupling makes our segmentation network robust to noise on an image. For fast computation, a segmentation algorithm is abstracted from the underlying oscillatory dynamics, and has been applied to synthetic and real images. Simulation results demonstrate the effectiveness of our oscillator network in image segmentation.
\end{abstract}

\section{INTRODUCTION}

One of the fundamental tasks in vision is image segmentation. It is the process to partition an image into a number of coherent regions; the pixels belonging to the same region should have similar properties in terms of intensity, color, and texture etc., while two adjacent regions should have dissimilar properties. Although humans perform the task without difficulty, image segmentation remains one of the most challenging problems in machine vision.

Neural networks have been successfully applied in many fields. However, little has been reported on image segmentation. Due to the lack of an efficient representational framework for encoding multiple objects simultaneously, image segmentation is particularly challenging for neural networks. Most of neural network methods pose image segmentation as pixel classification [1] and typically use a supervised learning process. Other segmentation methods include Boltzmann machines [2], multilayer perceptrons [3], and biologically motivated neural networks [4]. However, all of these methods achieve only limited success. Recently, Kohonen's self-organization maps have been also applied to segmentation [5]. A major shortcoming in this approach is that there is no autonomous control over the number of regions that should be segmented.

The discovery of synchronous oscillations in the brain supports the oscillatory representation to implement temporal correlation[6][7]; where each object is represented by a group of synchronized oscillators and different objects are represented by different groups whose oscillations are desynchronized from each other. Recently, Terman and Wang proposed a class of locally excitatory globally inhibitory oscillator networks (LEGION) on the basis of oscillatory correlation [8]. LEGION can rapidly achieve both synchronization and desynchronization, and has been successfully applied to real image segmentation [9]. However, due to pair-wise coupling between oscillators, the LEGION network is sensitive to noise; for example, it does not work for the noisy image in Figure 3(a).

In this paper, we propose a dynamically coupled network on the basis of oscillatory correlation and LEGION. We use an ensemble of oscillators in a local region for grouping instead of pair-wise coupling. Dynamical coupling is proposed to generate a local coupling structure for an oscillator in terms of its surrounding active oscillators, and a local coupling structure provides the basis for computing local coherent properties of an object. Two grouping rules are proposed for examining the similarity between oscillators, which leads to robust performance for image segmentation. For fast computation, we extract a segmentation algorithm from oscillatory dynamics. The algorithm has been successfully applied to both synthetic and real images.

In the next section we describe the proposed oscillator network, including dynamical coupling structures and grouping rules. In Section III, an algorithm derived from oscillatory dynamics is presented for gray-level image segmentation. Section IV reports the segmentation results on both synthetic and real images. Further discussions are given in Section $\mathrm{V}$.

\section{MODEL DESCRIPTION}

In our neural oscillator network, a single oscillator $(i, j)$ consists of an excitatory unit $x_{i j}$ and an inhibitory unit $y_{i j}$ as defined by Wang and Terman [9]:

$$
\begin{gathered}
\dot{x}_{i j}=3 x_{i j}-x_{i j}^{3}+2-y_{i j}+I_{i j} H\left(p_{i j}-\theta\right)+S_{i j}+\rho, \\
\dot{y}_{i j}=\epsilon\left[\alpha\left(1+\tanh \left(\beta x_{i j}\right)\right)-y_{i j}\right] .
\end{gathered}
$$

Here $H(\cdot)$ is the Heaviside step function defined as $H(\nu)=$ 1 if $\nu \geq 0$ and $H(\nu)=0$ if $\nu<0 . I_{i j}$ denotes the external stimulation to the oscillator, and $S_{i j}$ represents overall coupling from other oscillators in the network. The parameter $\rho$ denotes the amplitude of a Gaussian noise term, which is introduced to test the robustness of the system and to actively assist in desynchronizing different patterns. The parameter $\epsilon$ is chosen to be small, $0<\epsilon \ll 1$. The dynamics of a single relaxation oscillator is summarized as follows, where we drop all the subscripts to simplify the presentation. The $x$-nullcline of $(1), \dot{x}=0$, is a cubic function, while the $y$-nullcline, $\dot{y}=0$, is a sigmoid function. The parameter $\beta$ controls the steepness of the sigmoid function and is chosen to be large. For an input $I>0$, the two nullclines intersect only on the middle branch of the cubic, and 
(1) gives rise to a stable periodic trajectory. In this case, the oscillator is called enabled and four bounding points for the periodic trajectory are $L C, L K, R K$, and $R C$ [9], whose $x$ values are $L C_{x}=-2, L K_{x}=-1, R K_{x}=1$, and $R C_{x}=2$, respectively. The periodic solution alternates between an active phase $\left(R K_{x} \leq x \leq R C_{x}\right)$ and a silent phase ( $L C_{x} \leq x \leq L K_{x}$ ). When the oscillator stays in the active phase, it is called active; otherwise, it is called silent. Within either phase, the oscillator exhibits near steady state behavior. In contrast, the transition between the two phases takes place on a fast time scale, referred to as jumping. The parameter $\alpha$ is introduced to control the relative times that the oscillator stays in the two phases, with a larger $\alpha$ implying a shorter active phase. For an input $I<0$, the two nullclines intersect on the left branch of the cubic, and (1) produces a stable fixed point. In this case, the oscillator is called excitable.

In (1a), the Heaviside term provides a mechanism to distinguish between major regions and noisy fragments. Wang and Terman suggested that a major region must contain at least one oscillator, called a leader, which is located at the center of a large, homogeneous region [9]. A noisy fragment does not contain such an oscillator. Basically, a leader receives large lateral excitation from its neighborhood. The variable $p_{i j}$ denotes the lateral potential of oscillator $(i, j)$, through the threshold $\theta$, which critically determines whether oscillator $(i, j)$ is a leader.

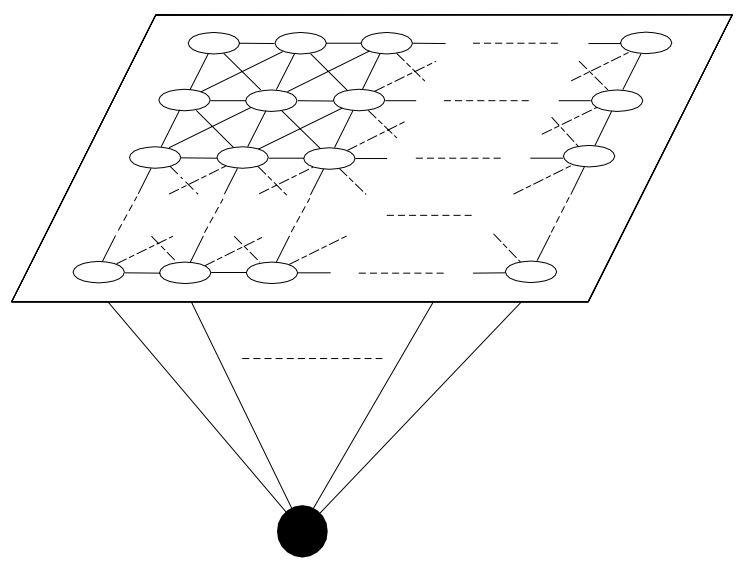

Figure 1. Architecture of a two-dimensional neural oscillator network. The global inhibitor is indicated by the black circle.

For image segmentation, we study two-dimensional oscillator networks. As illustrated in Figure 1, there is a local fixed structure, where an oscillator is connected to only eight immediate neighbors except for those on the boundaries where no wraparound is used. The activation of an oscillator spreads through fixed connections only. The global inhibitor is connected with all the oscillators in the twodimensional network, and its activity, $z$, is defined as

$$
\dot{z}=\phi\left(\sigma_{\infty}-z\right),
$$

where $\sigma_{\infty}=0$ if $x_{i j}<\theta_{z}$ for every oscillator $(i, j)$, and $\sigma_{\infty}=1$ if $x_{i j} \geq \theta_{z}$ for at least one oscillator. $\theta_{z}$ is a threshold. If $\sigma_{\infty}=0$, the global inhibitor is not triggered and the oscillators on the network receive no inhibition from the global inhibitor. If $\sigma_{\infty}=1$, the global inhibitor is triggered and all the oscillators on the network receive inhibition. $\phi \gg \epsilon$ is a parameter.

In our model, oscillators that are in the active phase simultaneously form a single segment, and as a result they should correspond to a set of coherent pixels. Therefore, image attributes of these active oscillators provide a coherence measure of the entire group. In an image with considerable noise, group-wide measures are more robust than single pixel measures; the latter are used by Wang and Terman [9]. On the other hand, proximity is one of the most important grouping principles; remote pixels, even though they may be in the same segment, should contribute little to recruiting a particular pixel. Based on these considerations, we use the attributes of a local ensemble, called a neighborhood, of active oscillators for grouping.

How to determine the size and the shape of such a neighborhood for grouping? The neighborhood should not be uniform; for example, pixels near a boundary between different regions need to be treated differently than pixels in the interior of a region because discontinuity near a boundary causes local image attributes to change dramatically. In general, the interior of an image region must be treated differently, in terms of grouping, than areas near the region boundary. Within the interior of a region, a larger and more uniform neighborhood can be used, whereas near a region boundary the neighborhood should be tailored so that it does not cross different regions. In oscillatory correlation, this can be naturally achieved by limiting the neighborhood to the currently active oscillators because they correspond to the same region. To deal with these different situations, we introduce a set of dynamical coupling neighborhoods and propose two grouping rules for handling region interior and region boundary separately; these are all embodied in the overall coupling term, $S_{i j}$, in (1a).

We denote the fixed set of the eight immediate neighbors of oscillator $(i, j)$ as $N(i, j)$ (see Figure 1). To measure similarity for oscillators that lie inside a homogeneous region, we introduce a pair of neighborhoods, referred to as pixel neighborhood and region neighborhood, respectively. The pixel neighborhood is used to measure local image attributes of a single oscillator, while the region neighborhood is used to measure those of the active neighbors of the oscillator. The pixel neighborhood of oscillator $(i, j)$ is defined as

$$
N_{1}^{a}(i, j)=\left\{(k, l) \mid i-R_{a} \leq k \leq i+R_{a}, j-R_{a} \leq l \leq j+R_{a}\right\},
$$

where $R_{a}$ is a parameter that determines the size of the pixel neighborhood. An example is shown in Figure 3(a). While $N_{1}^{a}(i, j)$ is fixed, the corresponding region neighborhood is dynamical, defined in terms of currently active neighbors of $(i, j)$ :

$$
\begin{gathered}
N^{a}(i, j)=\{(k, l) \mid(k, l) \in \\
\qquad \begin{array}{ll}
(m, n) \in N(i, j), x_{m n} \geq 0, H\left(u_{m n}-0.5\right)=0 \\
\left.N_{1}^{a}(m, n)\right\},
\end{array} \\
N_{2}^{a}(i, j)= \begin{cases}N^{a}(i, j) & \left|N^{a}(i, j)\right|>0 \\
N_{2}^{b}(i, j) & \text { otherwise }\end{cases}
\end{gathered}
$$

Here $u_{m n}$ is a variable indicating whether the corresponding pixel of the active oscillator $(m, n)$ is near a boundary, 
to be defined later on. The above union operation is performed only over those active oscillators in $N(i, j)$ that are considered to be in the interior of a region. The motivation for this should be clear later when the second pair of neighborhoods are introduced for pixels that are near a region boundary. Figure 3B gives an example of the region neighborhood corresponding to $\left|N^{a}\right|>0 . N_{2}^{b}(i, j)$ is an alternative region neighborhood to be defined below. The region neighborhood provides a local measure of an image region being expanded.

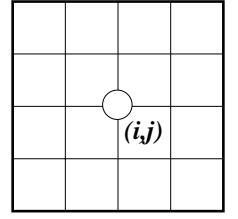

$N_{I}^{a}$

(a)

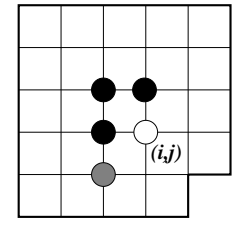

$N_{2}^{a}$

(b)

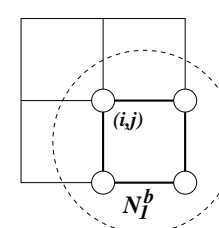

(c)

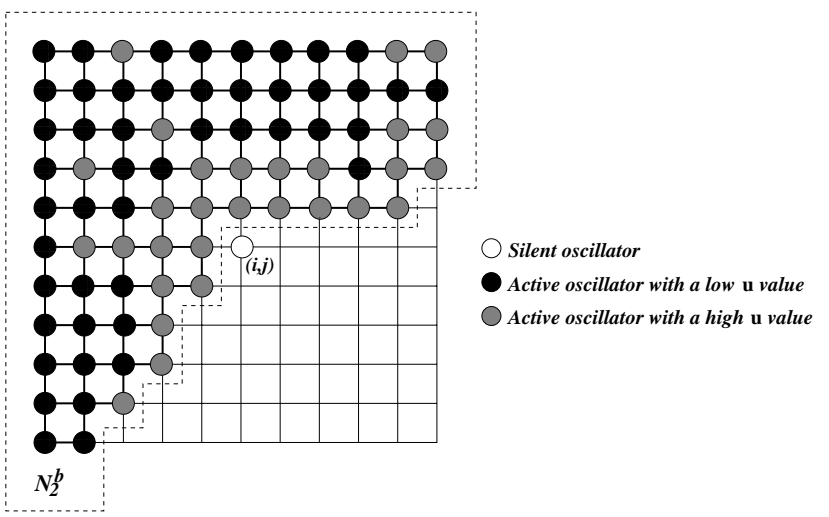

(d)

Figure 2. Examples of various neighborhoods. (a) The pixel neighborhood $N_{1}^{a}(i, j)$. (b) The region neighborhood $N_{2}^{a}(i, j)$. (c) The pixel neighborhood $N_{1}^{b}(i, j)$. (d) The region neighborhood $N_{2}^{b}(i, j)$.

To deal with those pixels near a region boundary separately, we introduce another pair of neighborhoods. The key for this pair is that neighborhoods should be limited to active oscillators only, since they belong to the same segment which corresponds to the same region. First, a pixel neighborhood of this kind is defined as

$$
N_{1}^{b}(i, j)=\left\{(k, l) \mid(k, l) \in N(i, j), x_{k l}<0\right\} \bigcup\{(i, j)\} .
$$

An example of this pixel neighborhood is illustrated in Figure 3C. Theoretically speaking, a single silent oscillator$(i, j)$ itself - may be evaluated for recruiting. However, as our experiments with real images show, single pixels are too noise-sensitive to obtain a reliable measure. That is why in the definition of $N_{1}^{b}(i, j)$ we include the silent neighbors of $(i, j)$. Notice that only silent oscillators should be included in $N_{1}^{b}$ because $(i, j)$ may belong to a region different from the currently expanding one. Finally, corresponding to $N_{1}^{b}$, a region neighborhood, $N_{2}^{b}(i, j)$, is defined as

$N_{2}^{b}(i, j)=\left\{(k, l) \mid i-R_{b} \leq k \leq i+R_{b}, j-R_{b} \leq l \leq j+R_{b}, x_{k l} \geq 0\right\}$.
Here the integer $R_{b}$ determines the size of the region neighborhood, and satisfies the following condition: $R_{b}$ is the minimal value guaranteeing that the number of active oscillators in $N_{2}^{b}(i, j)$ is no less than $\left(2 R_{0}+1\right)^{2} / 2$, where $R_{0}$ is a pre-specified constant, and $R_{b} \leq[\min (M, N) / 2]$ assuming that the size of the image is $M \times N$. Figure $3 \mathrm{D}$ gives an example of $N_{2}^{b}(i, j)$. Compared to a typical $N_{1}^{b}(i, j)$, which includes the neighborhoods of active oscillators, $N_{2}^{b}(i, j)$ includes only active oscillators themselves. This reflects the fact that we use $N_{2}^{b}$ for grouping pixels near a region boundary, and a neighborhood of an active oscillator is likely to cross different regions. The latter situation will produce unreliable measures and thus should be avoided.

All the neighborhoods in (3)-(6) are defined without explicitly considering the boundary of the entire image; and they are delimited by the boundary. Except for $N_{1}^{a}(i, j)$, shapes of all the neighborhoods defined above are dynamically determined by all the active oscillators surrounding a specified silent oscillator $(i, j)$ and specify dynamical coupling structures associated with oscillator $(i, j)$. Such dynamical coupling structures are incorporated by the grouping rules described later to yield robust segmentation performance.

To detect homogeneity in gray-level images, the first- and the second-order statistics are commonly used. Similarly, we adopt mean, $\mu$, and standard deviation, $\sigma$, to measure homogeneity in a specific neighborhood. Note that the mean and standard deviation are set to zero if the neighborhood is the empty set. Based on the similarity principle, grouping takes place if the local attributes of a silent oscillator are similar to those of a local region corresponding to a set of local active oscillators. To detect the similarity, we suggest two grouping rules for the two situations considered above: region interior and region boundary. First, we examine similarity for the type a pair of neighborhoods, $N_{1}^{a}$ and $N_{2}^{a}$. For oscillator $(i, j)$, we have

$$
\begin{aligned}
& S_{\mu}^{a}(i, j)=\omega_{\mu}^{a}-\left|\mu_{N_{1}^{a}(i, j)}-\mu_{N_{2}^{a}(i, j)}\right|, \\
& S_{\sigma}^{a}(i, j)=\omega_{\sigma}^{a}-\left|\sigma_{N_{1}^{a}(i, j)}-\sigma_{N_{2}^{a}(i, j)}\right| .
\end{aligned}
$$

Here $\omega_{\mu}^{a}$ and $\omega_{\sigma}^{a}$ are two tolerance parameters for measuring similarity. The subscripts, $N_{1}^{a}(i, j)$ and $N_{2}^{a}(i, j)$, indicate where the statistics are calculated. $S_{\mu}^{a}(i, j)$ and $S_{\sigma}^{a}(i, j)$ are measures in terms of the mean and the standard deviation. Similarly, we examine similarity for the type $b$ pair of neighborhoods, $N_{1}^{b}$ and $N_{2}^{b}$, as follows:

$$
\begin{aligned}
& S_{\mu}^{b}(i, j)=\omega_{\mu}^{b}-\left|\mu_{N_{1}^{b}(i, j)}-\mu_{N_{2}^{b}(i, j)}\right|, \\
& S_{\sigma}^{b}(i, j)=\omega_{\sigma}^{b}-\left|\sigma_{N_{1}^{b}(i, j)}-\sigma_{N_{2}^{b}(i, j)}\right| .
\end{aligned}
$$

Based on (7) and (8), we formulate two grouping rules for oscillator $(i, j)$ with the condition that there is at least one active oscillator in $N(i, j)$ : (a) the oscillator will be recruited if $S_{\mu}^{a}(i, j) \geq 0$ and $S_{\sigma}^{a}(i, j) \geq 0$; (b) the oscillator is recruited if $S_{\mu}^{b}(i, j) \geq 0$ and $S_{\sigma}^{b}(i, j) \geq 0$. The two grouping rules recruit $(i, j)$ in two different ways, and together implement both the proximity and the similarity principles. When an oscillator lies in a homogeneous region, Rule $a$ should be used for recruitment. In contrast, when it is near a boundary between different regions, Rule $a$ is unlikely to 
yield recruitment and Rule $b$ is used instead for expanding the region to produce a precise region boundary.

We now define $u_{i j}$ introduced in (4) and want $u_{i j}$ to approach unity only if oscillator $(i, j)$ is activated by Rule $b$ rather than Rule $a$. Therefore, $u_{i j}$ is defined as

$$
\dot{u}_{i j}=\lambda\left(\delta_{i j}^{b}-u_{i j}\right) H\left(\dot{x}_{i j}-\eta\right)-\epsilon u_{i j},
$$

where $\delta_{i j}^{b}=\left[1-H\left(S_{\mu}^{a}(i, j)\right) H\left(S_{\sigma}^{a}(i, j)\right)\right] H\left(S_{\mu}^{b}(i, j)\right) H\left(S_{\sigma}^{b}(i, j)\right)$. $\lambda \gg \epsilon$ is a parameter indicating the rate at which $u_{i j}$ changes. The Heaviside function, within which $\eta$ is a parameter, dictates that $u_{i j}$ does not change on the fast time scale unless the derivative of $x_{i j}$ increases significantly; in other words, $u_{i j}$ does not change much unless the corresponding oscillator jumps up.

The initial value of $u_{i j}$ is set to zero. Thus, according to (9) $u_{i j}$ stays at zero unless only Rule $b$ is used in recruiting $(i, j)$. In the latter case, $u_{i j}$ approaches one quickly. Beyond a brief time of jumping up, $u_{i j}$ slowly decays to zero since the first term in (9) is zero, and the second term acts on the slow time scale. The slow decay of $u_{i j}$ ensures that once it is activated it stays at a high value for a while, say for the time period of the active phase. Taken together, $u_{i j}$ serves the purpose of recording whether $(i, j)$ has been recruited in the last cycle of oscillation by Rule $b$ only, or whether pixel $(i, j)$ is near a region boundary. Thus, (9) implements the requirement for $N_{2}^{a}(i, j)$, the only neighborhood that uses $u_{i j}$. Given that the pixels recruited by Rule $b$ correspond to near-boundary ones, it should now be clear that these pixels should be excluded in the definition of type $a$ neighborhoods, which are used to evaluate the recruiting of interior pixels of a region. $r$ On the basis of two grouping rules, the overall coupling term, $S_{i j}$, in (1a) is given by

$$
\begin{aligned}
S_{i j}= & {\left[H\left(S_{\mu}^{a}(i, j)\right) H\left(S_{\sigma}^{a}(i, j)\right)+H\left(S_{\mu}^{b}(i, j)\right) H\left(S_{\sigma}^{b}(i, j)\right)\right] } \\
& H\left(\sum_{(k, l) \in N(i, j)} H\left(x_{k l}\right)-0.5\right)-W_{z} H\left(z-\theta_{z}\right) .
\end{aligned}
$$

Here $W_{z}$ is the weight of inhibition from the global inhibitor $z$. In our network, both $W_{z}$ and $\theta_{z}$ are always set to 0.5 . Note that $S_{i j}$ depends upon fixed connectivity, and there must be at least one active oscillator in $N(i, j)$ in order for $(i, j)$ to be recruited. This yields a connectivity constraint. Under this condition, when either of two grouping rules is satisfied, $S_{i j}>0$; otherwise, $S_{i j}<0$ due to the inhibition.

We summarize the dynamics of our network as follows. Suppose that all the oscillators are initialized in the silent phase. Once a leader enters the active phase, it triggers the global inhibitor, which exerts inhibition on the entire network. For a silent oscillator, its overall coupling is positive if either Rule $a$ or $b$ is satisfied, and in this case the oscillator jumps to the active phase. Otherwise, overall coupling is negative due to the global inhibition, which makes the oscillator stay in the silent phase. In this way, activation is propagated to other oscillators via local fixed connectivity until all the oscillators representing the same region are activated. Thus, the dynamics of our network preserves the property of LEGION. That is, it is the process of both synchronization through local excitation among neighboring oscillators and desynchronization through global inhibition [8][9].
Given that the emphasis of this paper is the notion of dynamical coupling and its effects in improving segmentation results, our following exposition focuses on a segmentation algorithm extracted from the dynamics of the oscillator network, which is needed for processing large images.

\section{Segmentation Algorithm}

Similar to Wang and Terman [9], we extract a segmentation algorithm from the dynamics of our network to reduce numerical computation. Basically, the algorithm preserves the essential properties of relaxation oscillators, e.g. two time scales, and network dynamics, e.g. synchronization and desynchronization.

For computational efficiency, we make several approximations to the dynamics of our network in the segmentation algorithm, as similarly done by Wang and Terman [9]. These include the selection of leaders, one time step to jump an enable oscillator, one time step for alternation between the active phase and the silent phase, and simultaneous jumping down all of the active oscillators belonging to the same region. Computation of overall coupling in (10) can be simplified as follows: Rule $a$ is first evaluated and only if its conditions are unsatisfied Rule $b$ is further evaluated. In this way, Rule $a$ has precedence and Rule $b$ may not need to be evaluated. The dynamical coupling structures are also generated accordingly. Similarly, we can also simplify the computation of $u_{i j}$. As mentioned before, the variable is used to distinguish between two classes of active oscillators. Therefore, $u_{i j}$ is only useful when oscillator $(i, j)$ is in the active phase. In our algorithm, $u_{i j}$ is treated as binary and initialized to zero, and it is set to unity once a silent oscillator is activated only by Rule $b$.

Based on the lateral potential concept [9], here we use an alternative method to select leaders for our network. We observe that oscillators near the center of a homogeneous region tend to have a high lateral potential. Based on this observation, we calculate the standard deviation on a fixed neighborhood defined as

$N_{p}(i, j)=\left\{(k, l) \mid i-R_{p} \leq k \leq i+R_{p}, j-R_{p} \leq l \leq j+R_{p}\right\}$,

where $R_{p}$ determines the size of the neighborhood. Oscillator $(i, j)$ is designated as a leader if and only if $\sigma_{N_{p}(i, j)} \leq$ $T_{p}$, where $T_{p}$ is a threshold. Intuitively, a larger value of $R_{p}$ results in leaders that are generated from larger homogeneous regions, while a smaller value of $T_{p}$ results in leaders from more homogeneous regions.

All the parameters, including $R_{p}$ and $T_{p}$ for selecting leaders, $R_{a}$ and $R_{0}$ for determining pixel and region neighborhoods, and the tolerance parameters, $\omega_{\mu}^{a}, \omega_{\sigma}^{a}, \omega_{\mu}^{b}, \omega_{\sigma}^{b}$, for testing the similarity, are specified in advance. The algorithm is summarized as follows:

1. Initialization

(a) Set $z(0)=0$.

(b) For every oscillator $(i, j)$ in the network, set $u_{i j}(0)=0$.

(c) Place all the oscillators randomly in the silent phase so that for every oscillator $(i, j), L C_{x}<x_{i j}(0)<L K_{x}$.

(d) Find leaders: $p_{i j}=H\left(T_{p}-\sigma_{N_{p}(i, j)}\right)$.

2. Find one oscillator $(i, j)$ satisfying the following conditions: (a) $p_{i j}=1$; (b) $x_{i j}(t) \geq x_{k l}(t)$, where oscillator $(k, l)$ in the silent phase and $p_{k l}=1$. Then,

$$
x_{i j}(t+1)=R K_{x} ; z(t+1)=1
$$




$$
\begin{aligned}
x_{k l}(t+1)=x_{k l}(t)+\left(L K_{x}-x_{i j}(t)\right), & \text { for }(k, l) \neq(i, j) \text { and } p_{k l}=1 .
\end{aligned}
$$

3. Iterate until all the leaders have been to the active phase.

If $\left(x_{i j}(t)=R K_{x}\right.$ and $\left.z(t)>z(t-1)\right)$,

$x_{i j}(t+1)=x_{i j}(t)$

else if $\left(x_{i j}(t)=R K_{x}\right.$ and $\left.z(t)<z(t-1)\right)$

$x_{i j}(t)=L C_{x} ; z(t+1)=z(t)-1$

else

$$
\text { If } z(t+1)=0 \text {, go to step } 2 .
$$

Form $N_{2}^{a}(i, j)$; Evaluate $S_{i j}(t+1)$ using Rule $a$

If $S_{i j}(t+1)>0$

else

$x_{i j}(t+1)=R K_{x} ; z(t+1)=z(t)+1$

Form $N_{1}^{b}(i, j)$ and $N_{2}^{b}(i, j)$;

Evaluate $S_{i j}(t+1)$ using Rule $b$

If $S_{i j}(t+1)>0$

$x_{i j}(t+1)=R K_{x}$

$z(t+1)=z(t)+1$

else

$u_{i j}(t+1)=1$

$x_{i j}(t+1)=x_{i j}(t)$

\section{Simulation Results}

We have applied our algorithm to many synthetic and real images [10]. Due to limited space, we report some typical results here.

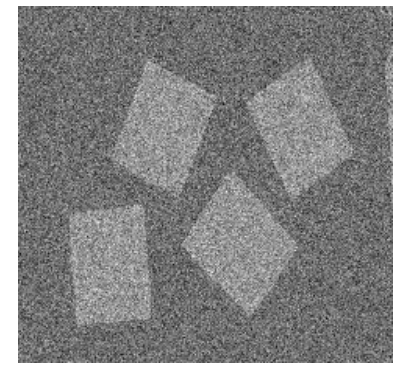

(a)

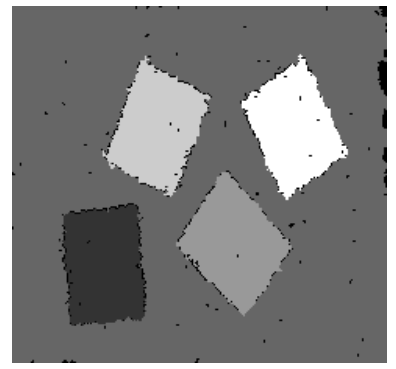

(b)
Figure 3. Segmentation result for a synthetic image. The parameters used in this simulation are $R_{p}=7, T_{p}=12.0$, $R_{a}=5, R_{0}=7, \omega_{\mu}^{a}=2.5, \omega_{\sigma}^{a}=4.0, \omega_{\mu}^{b}=3.0$, and $\omega_{\sigma}^{b}=4.5$. (a) Original image consisting of $230 \times 240$ pixels. (b) A gray map showing the entire segmentation result.

We first apply our algorithm to a typical noisy synthetic image illustrated in Figure 3(a), which was used by Sarkar and Boyer [11]. The image contains four parallelograms and an image background. Due to substantial noise, the LEGION algorithm [9] fails to segment the image into five meaningful regions. This is because their segmentation is based on pair-wise coupling within a fixed neighborhood (eight nearest-neighbor coupling). As a result, grouping is vulnerable to noise. When applied to Figure 3(a), their algorithm shows two kinds of error: breaks a region into fragments and conjoins regions with the image background. In the simulation using our algorithm, an oscillator network consisting of $230 \times 240$ oscillators and a global inhibitor is used so that each oscillator corresponds to one pixel in the image. After the image is presented, the algorithm quickly produces segregated regions at different time steps. To display the complete scenario of segmentation, we use the socalled gray map convention, where each gray level indicates a distinct segment and all the excitable oscillators constitute the background corresponding to those parts with high intensity variations [9]. Figure 3(b) is the resulting gray map of this simulation, where the background is indicated by the tiny black scattered areas. It is evident that our algorithm segments the noisy image into five meaningful regions.

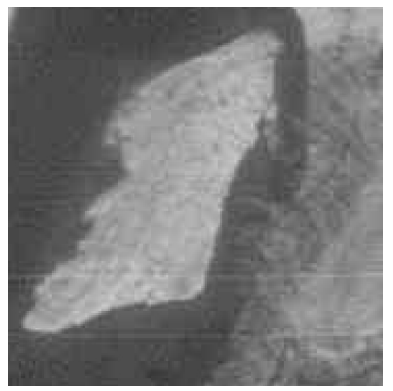

(a)

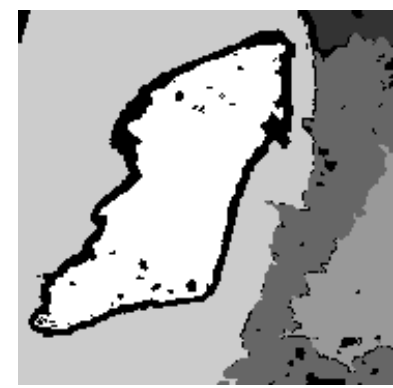

(b)
Figure 4. Segmentation result for an aerial image. The parameters used in this simulation are $R_{p}=5, T_{p}=7.0$, $R_{a}=2, R_{0}=5, \omega_{\mu}^{a}=2.0, \omega_{\sigma}^{a}=3.8, \omega_{\mu}^{b}=3.5$, and $\omega_{\sigma}^{b}=4.8$. (a) Original image consisting of $181 \times 181$ pixels. (b) A gray map showing the entire segmentation result.

The next image that our algorithm is tested on is an aerial image shown in Figure 4(a), a portion of a large aerial image to be used later. There are several objects, including water body, forest, island, and land. Shortly after the image is presented to our network, five segments pop out at different times. The five segments correspond to the island, the water body, the forest, and two parts of land. Figure 4(b) shows the entire segmentation result by a gray map, where the background is indicated by the black areas. Our algorithm successfully segments the noisy image into the meaningful regions.

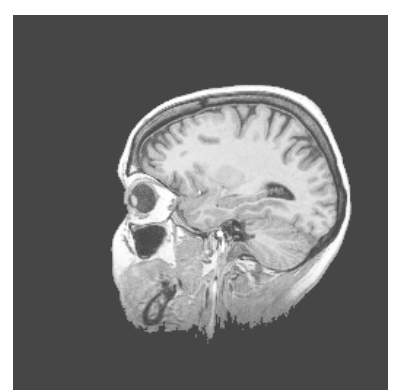

(a)

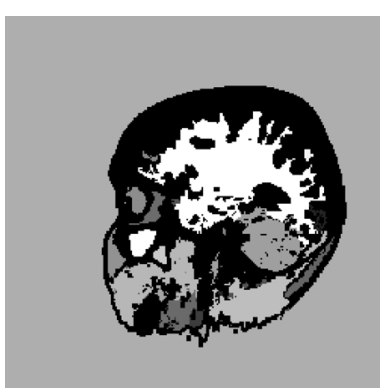

(b)
Figure 5. Segmentation result for an aerial image. The parameters used in this simulation are $R_{p}=3, T_{p}=5.0$, $R_{a}=1, R_{0}=4, \omega_{\mu}^{a}=2.2, \omega_{\sigma}^{a}=2.5, \omega_{\mu}^{b}=3.0$, and $\omega_{\sigma}^{b}=3.1$. (a) Original image consisting of $257 \times 257$ pixels. (b) A gray map showing the entire segmentation result.

We use an MRI image of human heads to further test our algorithm. Figure 5(a) is a midsagittal MRI image, and our algorithm segments the image into 28 regions plus a background, as shown in the gray map of Figure 7(b). Many salient regions are separated such as the cortex, the cerebellum, the lateral ventricle, the eye ball, and the sinus. In 
particular, the cerebellum is segregated with good accuracy though it has a low contrast with the adjacent cortex. The MRI image has been used to test the original LEGION algorithm [9], and our segmentation result is better. Our segments do not contain 'pepper and salt' like holes. Also, the cerebellum is segmented better for Figure 5(a) in our result than in theirs.

We have compared our network with several recent image processing methods in segmenting the above images [10]. These methods include a multiscale adaptive segmentation method [12] and several nonlinear smoothing methods [13] to remove noise prior to segmentation. Due to limited space, we give only a synopsis here. Our proposed network outperforms the multiscale adaptive segmentation method in general. The multiscale method either fails to segment these images into meaningful regions or produces over-segmentation. On the other hand, our comparisons show that an effective nonlinear smoothing technique plus subsequent segmentation, e.g. by the region growing algorithm, yields results comparable to ours. However, our network yields more precise water body boundaries in Figure 4. Note that nonlinear smoothing suffers from the termination problem [13], which generally requires human intervention to select the best results.

\section{DISCUSSION}

The major difference between our network and the LEGION network of Wang and Terman [9] lies in how to determine overall coupling, $S_{i j}$, in (1a). For gray-level image segmentation, pair-wise coupling is used in their network so that the coupling strength between two neighboring oscillators is determined by the similarity of two corresponding pixels [9]. Such pair-wise coupling leads to a fixed coupling structure. Unlike their network, we adopt dynamical coupling structures and use two grouping rules to recruit a silent oscillator in two different ways. Thus, our network has the property that the labeling of an oscillator is considered at any time through two grouping rules on the basis of dynamical coupling structures. This property gives rise to robust performance, and does not exist in their network. On the other hand, both their network and our network share the property of local coupling that plays a fundamental role in image segmentation. As pointed out in Section 2, our network shares the same properties of LEGION dynamics that has been extensively analyzed by Terman and Wang [8], e.g. fast synchronization within an oscillator group representing each object and desynchronization among oscillator groups representing different objects. Therefore, our neural oscillator network preserves biological plausibility of LEGION argued by Wang and Terman [9].

A salient feature of our network is its robust performance in noisy image segmentation. Noise is unavoidable in real image processing. In order to achieve good segmentation performance, a smoothing technique is often employed for noise removal prior to the use of a segmentation method, which results in a two-stage segmentation approach. In general, smoothing techniques can be classified into two categories: linear and nonlinear smoothing. However, both of them have different problems in practice. Linear smoothing causes important discontinuities to be blurred so that meaningful objects cannot be separated during segmenta- tion. The general idea behind nonlinear smoothing is to adapt pixel values to the local properties of an image. Although the use of nonlinear smoothing avoids, to a good extent, the problem existing in linear smoothing, it causes the termination problem [13]. Unlike the two-stage segmentation approach, in our network no iterative smoothing operation is involved so that the termination problem does not occur. This feature distinguishes our approach from those two-stage segmentation methods.

To conclude, we have presented a new method to image segmentation, which is based on dynamical coupling and oscillatory correlation. Our study demonstrates that robust segmentation performance can be achieved based on the idea of using dynamical neighborhoods, which is inherently consistent with the dynamics of relaxation oscillators. Our results lend further support to oscillatory correlation as a potential solution to scene segmentation and perceptual organization (see also [9]).

\section{ACKNOWLEDGMENTS}

This work was done while $\mathrm{KC}$ was a visiting scientist at OSU. Authors are grateful to X. W. Liu for discussions. This work was supported in part by an NSF grant (IRI9423312), an ONR grant (N00014-93-10335) and an ONR Young Investigator Award (N00014-96-1-0676) to DLW.

\section{REFERENCES}

[1] N. R. Pal and S. K. Pal, "A review on image segmentation techniques," Pattern Recogn. 26, 1993, 1277-1294.

[2] T. J. Sejnowski and G. E. Hinton, "Separating figure from ground with a Boltzmann machine," In Vision, Brain, and Cooperative Computation, M. A. Arbib \& A. R. Hanson Eds., 1987, 703-724.

[3] M. C. Mozer, R. S. Zemel, M. Behrmann, and C. K. I. Williams, "Learning to segment images using dynamic feature binding," $\mathrm{Neu}$ ral Comp. 4, 1992, 650-665. 1988, 52-59.

[4] S. Grossberg and L. Wyse, "A neural network architecture for figure-ground separation of connected scenic figures," Neural Net., 4, 1991, 723-742.

[5] S. M. Bhandarkar, J. Koh, and M. Suk, "Multiscale image segmentation using a hierarchical self-organizing map," Neurocomputing 14, 1997, 241-272.

[6] P. Milner, "A model for visual shape recognition," Psychol. Rev. 81, 1974, 512-535.

[7] von der Malsburg, "The correlation theory of brain function," Inter nal Report 81-2, Max-Planck-Institute for Biophysical Chemistry, 1981.

[8] D. Terman and D. L. Wang, "Global competition and local cooperation in a network of neural oscillators," Physica D 81, 1995, 148176.

[9] D. L. Wang and D. Terman, "Image segmentation based on oscillatory correlation," Neural Comp. 9, 1997, 805-836. (for errata, see Neural Comp., vol. 9, pp. 1623-1626)

[10] K. Chen and D. L. Wang, "A dynamically coupled neural oscillator network for image segmentation," Technical Report OSU-CISRC7/98-TR24, Department of Computer and Information Science, The Ohio State University, USA.

[11] S. Sarkar and K. L. Boyer, "On optimal infinite impulse response edge detection filters," IEEE Trans. Pattern Anal. Machine Intell. 13, 1991, 1154-1171.

[12] T. Caelli and W. Bischof, Machine learning and image interpretation, New York: Plenum, 1997.

[13] J. Weickert, "A review of nonlinear diffusion filtering," In ScaleSpace Theory in Computer Vision, B. Romeny, L. Florack, J. Koenderink, \& M. Viergever Eds., 1997, 3-28. 